\title{
Inference of Animal Activity From GPS Collar Data on Free-Ranging Cattle
}

\author{
Eugene D. Ungar, ${ }^{1}$ Zalmen Henkin, ${ }^{2}$ Mario Gutman, ${ }^{1}$ \\ Amit Dolev, ${ }^{3}$ Avraham Genizi, ${ }^{4}$ and David Ganskopp ${ }^{5}$

\begin{abstract}
Authors are ${ }^{1}$ Research Scientists, Department of Agronomy and Natural Resources, Institute of Field and Garden Crops, Agricultural Research Organization, the Volcani Center, P. O. Box 6, Bet Dagan 50250, Israel; ${ }^{2}$ Research Scientist and ${ }^{3}$ graduate student, MIGAL - Galilee Technology Center, P. O. Box 831, Qiryat Shemona, 11016, Israel; ${ }^{4}$ Statistician, and ${ }^{5}$ Rangeland Scientist, USDA Agricultural Research Service, Eastern Oregon Agricultural Research Center, 67826-A Hwy 205, Burns, OR 97720.
\end{abstract} \\ Department of Statistics and Experiment Design, Agricultural Research Organization, the Volcani Center, Bet Dagan, Israel;
}

\begin{abstract}
Global positioning systems (GPSs) enable continuous and automatic tracking of an animal's position. The value of such spatialtemporal information can be improved if the corresponding activity of the animal is known. We evaluated the potential of Lotek GPS collars to predict activity of beef cattle on extensive rangeland in 2 contrasting foraging environments. Collars were configured to record animal location at intervals of 20 minutes (United States) or 5 minutes (Israel), together with counts from 2 motion sensors. Synchronized field observations of collared cows were conducted in 1999 (United States) and in 2002 and 2003 (Israel). Grazing, traveling (without grazing), and resting activities were recorded as minutes out of 20 for each category (United States), or as a single category (Israel). For the US data, stepwise regression models of grazing, traveling, and resting time accounted for $74 \%-84 \%$ of the variation, on the basis of the motion sensor counts for the left-right axis and the distances between GPS fixes. Regression tree analysis of grazing time yielded a simple model ( 4 splits) that accounted for $85 \%$ of the variation. For the Israeli data, the misclassification rates obtained by discriminant analysis and classification tree analysis of animal activity were $14 \%$ and $12 \%$, respectively. In both analyses, almost all grazing observations were correctly classified, but other activities were sometimes misclassified as grazing. Distance alone is a poor indicator of animal activity, but grazing, traveling, and resting activities of free-ranging cattle can be inferred with reasonable accuracy from data provided by Lotek GPS collars.
\end{abstract}

\section{Resumen}

Los sistemas de posicionamiento global (GPS) permiten el rastreo continuo y automático de la posición de un animal. El valor de tal información espacial-temporal pueden ser mejorado si la actividad correspondiente del animal es conocida. Evaluamos el potencial de los collares de GPS Lotek para predecir la actividad del ganado para carne en un pastizal extensivo en dos ambientes de forrajeo contrastantes. Los collares fueron configurados para registrar la ubicación del animal a intervalos de $20 \mathrm{~min}$ (US) o 5 min (Israel), junto con conteos de dos sensores de movimiento. En 1999 (US), 2001 y 2002 (Israel) se condujeron observaciones de campo sincronizadas de las vacas con collar. Se registraron actividades de apacentamiento, viaje (sin apacentamiento) y descanso en términos de minutos dedicados a cada actividad dentro del período de 20 minutos (US) o como una sola categoría (Israel). Para los datos de US, los modelos de regresión a pasos para apacentamiento, viaje y tiempo de descanso explicaron de $74-84 \%$ de la variación, basados en los conteos del sensor de movimiento para los ejes derecho-izquierdo y las distancias entre las determinaciones del GPS. El análisis de regresión de árbol del tiempo de apacentamiento produjo un modelo simple (cuatro divisiones) que explicaron el $85 \%$ de la variación. Para los datos de Israel, las tasas de clasificación errónea obtenidas por el análisis discriminante y el análisis de clasificación de árbol fueron $14 \%$ y $12 \%$ respectivamente. En ambos análisis, casi todas las observaciones de apacentamiento fueron clasificadas correctamente, pero las otras actividades algunas veces fueron clasificadas erróneamente como apacentamiento. La distancia sola es un indicador pobre de la actividad animal, pero las actividades de apacentamiento, viaje y descanso del ganado en libre apacentamiento pueden ser inferidas con una certeza razonable a partir de datos provenientes de collares de GPS Lotek.

Key Words: global positioning system, foraging behavior, grazing time, motion sensors, classification and regression trees

Research in Israel was funded by the Ministry of Agriculture (Chief Scientist and Rangelands Board) and Northern R\&D. Contribution from the Agricultural Research Organization, Institute of Field and Garden Crops, Bet Dagan, Israel. No. 124/2003.

Throughout this paper, presentation of trade names is for information only and does not constitute an endorsement of one product over another that may be suitable.

Correspondence: E. D. Ungar, Department of Agronomy and Natural Resources, Institute of Field and Garden Crops, Agricultural Research Organization, the Volcani Center, P. O. Box 6, Bet Dagan 50250, Israel. Email: eugene@volcani.agri.gov.il

Manuscript received 9 April 2004; manuscript accepted 27 December 2004.

\section{INTRODUCTION}

The study of landscape use by animals requires a record of the location of individuals over time. Technical and logistic difficulties in obtaining and collating such data have hampered the study of free-ranging animals in a spatially explicit way (Coughenour 1991). Tracking animals using the global positioning system represents a major advance in spatiotemporal data acquisition. 
Over the last decade, many species of terrestrial wildlife have been fitted with GPS collars. Examples are moose (Rodgers and Anson 1994; Rempel et al. 1995; Moen et al. 1996a, 1996b; Dussault et al. 1999; Girard et al. 2002), deer (Blanc and Brelurut 1997; Merrill et al. 1998; Bowman et al. 2000), elk (Biggs et al. 2001), caribou (Johnson et al. 2002a, 2002b), and elephant (Blake et al. 2001). GPS tracking collars have been incorporated in research on the ecology and management of grazing systems using sheep (Rutter et al. 1997; Hulbert et al. 1998) and cattle (Turner et al. 2000; Ganskopp 2001). By using GPS units in conjunction with geographic information systems (GISs), animal distribution and movement can be related to landscape features. For example, Ganskopp (2001) used this technology to evaluate the efficacies of salt and water manipulations for affecting cattle distribution.

Foraging ecology inferences can be made by pairing animal location data with associated animal activity data (Graham 2001). The greatest impact large herbivores have on the landscape is the removal of forage; therefore, a first priority is to determine where and for how long an animal has grazed a landscape element. Other activities of interest might include traveling (without grazing) and resting. In principle, activity might be inferred from the distances between successive GPS locations, with short, medium, and long distances corresponding to rest, grazing, and traveling, respectively. However, this approach may be less reliable than expected, given the inherent level of error in GPS locations when emphasis in collar design is speed of position acquisition (to conserve battery life) rather than accuracy. Variability of livestock movements may also confound such an approach.

Some commercially available GPS collars incorporate motion sensors that tally activations over a specified time period as indices of activity. The calibration of predictive models to discriminate among activities requires synchronized visual observations of collared individuals. Ganskopp (2001) utilized regression analyses to examine the relationships of observed animal activity to the linear distance between GPS fixes and motion-sensor counts. He concluded that activity did not correlate in a consistent and simple manner with distance and motion data. Schauer (2003) has suggested that the operational configuration of the collar and individual cow/collar fit may be important factors. Calibration data sets are not easily collected under extensive grazing conditions; therefore the development of robust generalized prediction models is important. We report results from 2 independent studies conducted to establish the relationships between GPS location data and activity in contrasting environments and with different operational configurations. Specific questions asked were: How well can animal activity be inferred from distances between location data alone? To what extent do motion-sensor data improve our predictive ability? What is the most appropriate predictive statistical model?

\section{MATERIALS AND METHODS}

\section{GPS Collars}

Synchronized GPS collar and observer data were gathered for free-grazing cattle in the United States and Israel. The observed animals wore Lotek GPS collars of the 2000 (United States) and 2200 (Israel) series (Lotek Engineering, Newmarket, ON,
Canada; see URL http://www.lotek.com). The collars can integrate and store a GPS fix at user-determined intervals, together with information from on-board temperature (ambient) and motion sensors.

Two captive-ball tilt switches (Comus International, Clifton, NJ, CW 1600 series) were incorporated into the collars to sense motion. Switches consist of a conducting ball inside a capsule that has a protruding contact pin inside the end. When the ball simultaneously contacts the sidewall and the pin, a circuit is completed and a count is registered. Acceleration and deceleration forces, as well as tilting, can cause the captive ball to move. The sensors are mounted in the collar at right angles to one another, with the long axes parallel to the ground. One sensor is most sensitive to left-right (activity 1), and the other to forward-backward movement (activity 2). This was confirmed using an upright collar by performing 100 movements of about $30 \mathrm{~cm}$ back and forth or left and right along a single axis of a level plain within 5-minute integration periods. Vertical sensitivity was assessed by lifting and lowering a suspended collar approximately the same distance. In the same examination, we confirmed that gentle up/down motions were not registered on either counter, whereas both sensors registered rapid shaking or twisting motions in any direction.

The motion sensors acquire data within a cyclic activitysampling period. The parameters for the activity-sampling period and the GPS fix interval, as well as the mode of operation of the motion sensors, are set by the user via a software interface. The GPS fix interval can range from 5 to $360 \mathrm{~min}$, and the activity-sampling period can be set between 1 and $60 \mathrm{~min}$ (but not greater than the GPS fix interval -1 ). The counters tally up to a maximum value of 255 . At the start of a GPS fix, the motion sensor counts in the left-right direction and in the fore-aft direction since the previous activity-sampling are stored and the system is reinitialized. Activity count data can be stored either as the left-right and fore-aft counts since the last completed activity-sampling period or as the mean counts of a number of activity-sampling periods since the start of the last GPS fix. In the latter case, the number of such periods $=$ integer (GPS fix interval - 1)/activity-sampling period.

On retrieval of a GPS collar from an animal, the coordinates of recorded positional fixes (in a latitude/longitude format) and accompanying data can be downloaded to a computer. Accompanying data include elevation, date, time, ambient temperature, the left-right and the fore-aft motion sensor counts, and satellite-related information.

\section{US Study}

Research was conducted at the Northern Great Basin Experimental Range (lat $119^{\circ} 43^{\prime} \mathrm{W}$, long $43^{\circ} 29^{\prime} \mathrm{N}$; elevation 1392 to $1674 \mathrm{~m}), 72 \mathrm{~km}$ west-southwest of Burns, Oregon. The mean annual temperature is $7.6^{\circ} \mathrm{C}$, with recorded extremes of $-29^{\circ} \mathrm{C}$ and $42^{\circ} \mathrm{C}$, and mean annual precipitation is $289 \mathrm{~mm}$ with about $60 \%$ of the accumulation falling as snow. The predominant soil at the experimental range is of the Ratto Series (clayey, montmorillonitic, frigid, shallow Xerollic Durargids). The vegetation is characterized by a tree layer of dispersed western juniper (Juniperus occidentalis Hook.), a shrub layer dominated by sagebrush species (Artemisia L.), and understory grasses including bluebunch wheatgrass (Agropyron spicatum (Pursh) 
Scribn. \& Smith), Idaho fescue (Festuca idahoensis Elmer), and Sandberg's bluegrass (Poa sandbergii Vasey).

Three pastures (825-859 ha) were used in this project. In 1999 each pasture was stocked with 40 Hereford X Angus cow/calf pairs from about 20 May to 20 September. On June 16 2 cows were selected at random from each pasture and fitted with Lotek 2000 GPS collars weighing about $1.15 \mathrm{~kg}$. The operational schedule of the collars used a GPS fix interval of 20 minutes and an activity-sampling period of 3 minutes. The left-right and fore-aft motion sensor counts stored with each GPS fix were averages for the 6 completed activity-sampling periods between GPS fixes. Sensor data acquired in the last 2 minutes of the 20-minute interval were not included in calculations of the mean. The 6 GPS collars were retrieved from the animals on $15 \mathrm{July}$, and 5 functioned correctly.

Visual observation of animal activity was conducted by two observers during daylight on 9 days from 18 June to 8 July 1999 using continuous sampling of focal animals (forage availability $>750 \mathrm{~kg} \mathrm{ha}^{-1}$ dry matter). For each of the 5 cows with functioning collars, 12-13 hours of observations were conducted over 2 ( 1 cow) or 3 ( 4 cows) days. Observers were able to maintain a distance of 20 to $50 \mathrm{~m}$ of focal cows without any noticeable change in behavior. Defined activities were grazing, traveling (walking or running without grazing), standing, lying, drinking water, and consuming trace-mineral salt. Activities were deemed exclusive, and even though cattle walk as they graze, grazing was considered to occur as long as the animal's head remained down. Fly control was accomplished with insecticide ear tags. Activities were recorded by technicians equipped with synchronized watches, using paper with rulerlike scales, each of which covered 1 hour at a 1-minute resolution. Data were compiled as the total number of minutes during which the animal was involved in each activity, within successive 20minute GPS fix intervals. The procedure was to observe the animal continuously and to note the precise time of any change in activity. If the new activity persisted for more than 30 seconds, its starting time was permanently marked and duration subsequently tracked. If the animal returned to its original activity within 30 seconds, the brief interlude was ignored.

The US study took place when the selective availability function of the GPS was still in force; therefore the location fixes were differentially corrected. Base station files were downloaded from a US Forest Service/Bureau of Land Management unit in Burns, Oregon (http://www.fs.fed.us/database/gps/burns.htm). Base station files and data were processed with Lotek N3Win1 software. GIS software (Idrisi V 2.005) was used to convert coordinates from latitude/longitude form to universal transverse Mercator (UTM 11-N) coordinates to facilitate algebraic derivation of straight-line distances between successive points.

\section{Israel Study}

Research was conducted on the Karei Deshe Experimental Range in the eastern Galilee region of Israel (lat $35^{\circ} 35^{\prime} \mathrm{E}$, long $32^{\circ} 55^{\prime} \mathrm{N}$; elevation $150 \mathrm{~m}$ ). Mean annual precipitation is $550 \mathrm{~mm}$, falling between October and April. Topography is hilly with a rock cover of about $30 \%$. Soil is a brown basaltic protogrumosol of variable depth, supporting a herbaceous plant community of over 250 species. Details of the vegetation at the site are given in Gutman and Seligman (1979) and Gutman et al. (1990).
Observations by 3 observers were conducted in 2 paddocks (28 ha each), which were stocked at 0.9 and 1.8 ha per cow, respectively, and were grazed from January or February through September. In 2002, from March to August, 2 Lotek 2200LR GPS collars (approximate weight $1.35 \mathrm{~kg}$ ) were deployed, 1 in each paddock; they were moved from one cow to another twice a week to increase the cow sample size. Daytime observations of 7 collared cows were conducted on 8 dates in June and July (forage availability $>2400 \mathrm{~kg} \mathrm{ha}^{-1}$ dry matter). During February to August 2003, 4 collars were deployed, 2 in each paddock. Collars were placed on randomly selected cows for 7 days and then placed on other cows. Four cows were observed on 6 dates in February (forage availability $>650 \mathrm{~kg} \mathrm{ha}^{-1}$ dry matter). In both years, the operational schedule of the collars used a GPS fix interval of 5 minutes and an activity-sampling interval of 4 minutes, without averaging of sensor counts. Observations were not recorded on days when cows were handled. Animals at the station are easily disturbed; therefore observations were conducted with binoculars from a distance of at least $50 \mathrm{~m}$. Activity was defined as grazing (including brief interludes of walking), traveling (without grazing), standing, and lying down. Activity transitions were recorded in the field, and from them we derived the activities during 5-minute GPS fix intervals, synchronized to the data from the GPS collars. A low proportion of 5-minute intervals $(<5 \%)$ contained more than one activity. These multipleactivity intervals were insufficient in number to be modeled, and were discarded subsequently from the analysis.

Collar data were not differentially corrected because of problems with the base station in the study area. Fortunately, observations in Israel took place after the GPS selective availability function had been disabled, which improved accuracy (Hulbert and French 2001; Adrados et al. 2002). GIS software (ArcView 3.2) was used to convert the coordinates from latitude/ longitude form to Israel local coordinates (local UTM net), to simplify the computation of distance between successive points.

\section{Analysis}

The United States and Israel observations yielded 195 and 233 data records, respectively, for analysis. Each record included: the straight-line two-dimensional distances (Distance, $\mathrm{m}$ ) traveled by the animal between adjacent GPS fixes, the left-right motion sensor count (CountLeftRight), the fore-aft motion sensor count (CountForeAft), and observed activities. The distance measures were probably underestimated because we assumed straight-line travel between adjacent points. Also, the last 1 (Israel) or 2 (United States) minutes of each activity observation are not represented in the motion sensor counts.

Statistical tools used to predict animal activity from GPS collar data were regression or discrimination, depending on whether the activity $(Y)$ was measured on a continuous scale (US data) or was divided into distinct classes (Israel data). We aimed for generality and parsimony in relationships rather than the best fit to the calibration data. One analysis was conducted with only the basic collar variables of the left-right motion sensor count, the fore-aft motion sensor count, and the distance as candidates for entry into the model, and a second analysis with the 9 two-way products and ratios of these 3 variables included as candidates for entry into the model. We used forward and 
Table 1. Overview of the observation data sets. In the United States, an activity was assigned to each minute of a 20 -min observation period. In Israel, a single activity was scored for each 5-min period.

\begin{tabular}{|c|c|c|c|c|c|c|c|c|c|}
\hline \multirow[b]{2}{*}{ Country } & \multirow[b]{2}{*}{ Animal } & \multicolumn{8}{|c|}{ Animal Activity } \\
\hline & & $n^{1}$ & G & $\mathrm{T}$ & S & L & W & M & Total \\
\hline & & & \multicolumn{7}{|c|}{ Minutes of Observation } \\
\hline \multicolumn{10}{|l|}{ United } \\
\hline \multirow[t]{7}{*}{ States } & 1 & 38 & 374 & 31 & 325 & 23 & 6 & 0 & 760 \\
\hline & 126 & 39 & 464 & 44 & 175 & 80 & 12 & 5 & 780 \\
\hline & 269 & 42 & 237 & 25 & 207 & 358 & 4 & 9 & 840 \\
\hline & 334 & 36 & 192 & 44 & 246 & 219 & 3 & 16 & 720 \\
\hline & 421 & 40 & 502 & 85 & 73 & 126 & 9 & 5 & 800 \\
\hline & Total & 195 & 1769 & 229 & 1026 & 806 & 34 & 35 & 3900 \\
\hline & & \multicolumn{8}{|c|}{ Number of 5 -min Observations } \\
\hline \multirow[t]{10}{*}{ Israel } & 26 & - & - & 3 & - & - & - & - & 3 \\
\hline & 46 & - & - & 5 & - & - & - & - & 5 \\
\hline & 58 & - & 12 & - & 8 & 12 & - & - & 32 \\
\hline & 78 & - & - & 3 & - & - & - & - & 3 \\
\hline & 117 & - & - & 5 & - & - & - & - & 5 \\
\hline & 130 & - & 7 & 3 & 26 & - & - & - & 36 \\
\hline & 134 & - & 24 & - & 18 & 20 & - & - & 62 \\
\hline & 394 & - & 37 & - & 2 & 16 & - & - & 55 \\
\hline & 813 & - & 15 & 4 & - & 11 & - & - & 30 \\
\hline & Total & - & 95 & 23 & 54 & 59 & - & - & 231 \\
\hline
\end{tabular}

${ }^{1} n$ in US data indicates number of 20-min periods sampled; G, grazing; $T$, traveling; S, standing; L, lying; W, drinking; M, mineral consumption.

backward stepwise procedures for multiple regression analyses, and simultaneous and stepwise procedures for the discrimination analyses. In the latter case we show Wilks' Lambda statistic, which is one of the principal statistics for testing the null hypothesis in multivariate analysis of variance. Wilks' Lambda test is a multivariate generalization of the univariate $F$ test, and tests the equality of the vector of means of the predicted variables in the different activities. If this test is significant, a useful discriminant function is expected to be found.

A cow/collar effect was tested by its inclusion in the analysis of variance of activity (grazing, resting, and traveling time), and by separate multiple regression analysis of activity for each cow/collar.

We also used the techniques of classification and regression trees (Breiman et al. 1984; Dahms 2004; Del Cima et al. 2004), as implemented in the Partition platform of the JMP statistical analysis program (Release 5.0.1a) (SAS Institute 2002). These techniques use the classifying variables to split the data into subgroups to achieve more homogeneity of $Y$. Homogeneity is expressed by the number of observations in the group having the same activity (classification trees), or the variance of $Y$ (regression). All splits are binary-the data is divided at the split point into two regions (greater than and less than); however, the same variable may be split repeatedly. In a regression tree, all observations in a subgroup receive a single predicted value (the number of minutes out of 20) for the activity being analyzed. In a classification tree, all observations in a subgroup receive a predicted activity (lasting $5 \mathrm{~min}$ ) equal to the activity with the highest frequency in the subgroup. An interactive, stepwise process of adding and pruning splits in the tree structure is used, to find a balance between model simplicity and predictive ability, while avoiding "overfitting"-introducing splits into the data that spuriously improve the fit. No assumptions are made regarding the underlying distribution of values of the predictor variables.

\section{RESULTS}

Table 1 provides an overview of the 2 data sets that were collected, according to animals and activity. In both countries, resting (a pooling of standing and lying) accounted for almost half of the observations. Grazing accounted for $45 \%$ and $41 \%$ of the observation time in the United States and Israel, respectively, and traveling for only $6 \%$ and $10 \%$ in the United States and Israel, respectively. Drinking and mineral consumption each accounted for less than $1 \%$ of the observation time (US data).

The frequency distributions of the left-right motion sensor count, the fore-aft motion sensor count, Distance (between GPS locations), and observed activity are shown in Figure 1 for the 2 study sites. A U-type distribution was exhibited by the left-right count (Figs. 1a and 1b). The proportions of left-right counts equal to 255, the maximum possible sensor count, were $9 \%$ and $14 \%$ in the United States and Israel, respectively. The fore-aft count and Distance variables showed i-type distributions (Figs. 1c-1f). A greater range of Distance was obtained in the United States (0-1 389 m; Fig. 1e) than in Israel (0-274 m; Fig. 1f), as was to be expected because cow location was recorded less frequently (20-vs. 5-minute intervals). Cattle in the United States spent a high proportion of time, within the 20-minute observations, occupied in a single activity (Fig. 1g). In $73 \%$ of observations, cattle grazed or rested for at least 16 minutes per observation. Traveling, drinking, and mineral consumption were recorded (i.e., occupied 1 or more minutes out of 20 ) in $19 \%, 7 \%$, and $3 \%$ of observations, respectively.

Figure 2 shows the 3 pairs of relationships between the basic collar variables. In the US data, observations that were dominated (at least 16 of 20 minutes) by grazing and resting separated out quite well according to the left-right count (Fig. 2a). Values less than approximately 100 were almost all resting. For the Israel data, a low left-right count (up to approximately 150) was associated with resting; however, both grazing and traveling appear to be associated with a high left-right count (Fig. 2b). Singularly, the straight-line distance between GPS locations (Distance) does not appear to be a good indicator of activity at either site (Figs. $2 \mathrm{c}$ and $2 \mathrm{~d}$ ). Certain regions of the response space of the 3 collar variables are occupied by a single activity. For example, a low left-right count in combination with low Distance (Figs. 2c and 2d) or a low left-right count in combination with a high fore-aft count (Figs. 2a and 2b) almost certainly indicates a resting activity, and large Distance values are almost certainly associated with traveling (Figs. 2d and $2 \mathrm{f}$ ) - but there are also regions of the response space that contain instances of all activities.

Figure 3 shows the relationships obtained in the United States between the number of minutes dedicated to an activity and the 3 collar variables. The low frequency of drinking activities (Figs. 3j-3l) and mineral consumption (Figs. 3m-3o) pre- 


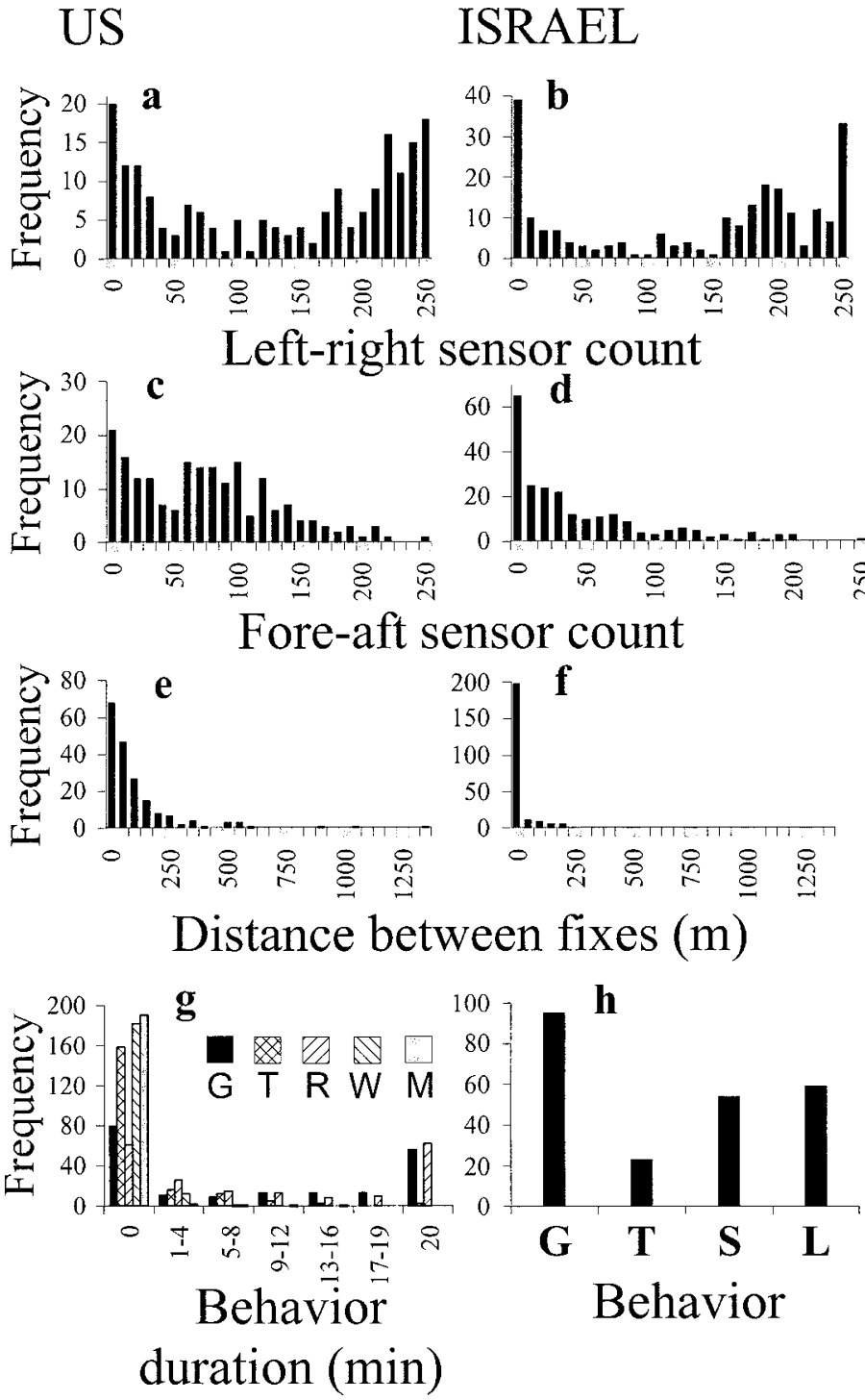

Figure 1. Frequency distributions of counts obtained from motion sensors detecting left-right and fore-aft movements, the straight-line distance between sequential GPS fixes, and animal activities, for the 2 study sites. Activities are in terms of the number of minutes of a 20minute observation period for the United States, and complete 5-minute periods for Israel. Activities are: G, grazing; $T$, traveling; $S$, standing; $\mathrm{L}$, lying; $\mathrm{R}$, resting; $(S+L), W$, drinking water; $M$, mineral consumption. Sensor count summations are for 3-minute (US) and 4-minute (Israel) periods.

cludes development of a predictive model for these activities. The number of minutes within a 20-minute observation dedicated to traveling was generally less than 10, although a relationship between traveling and Distance is apparent (Fig. 3f). The bulk of the data, consequently, consisted of grazing and resting activities. The proportion of observation time spent grazing or resting appears to be most clearly related to the leftright count, where response to grazing $(r=0.86, P<0.0001$; Fig. 3a) was inverse to that of resting $(r=-0.91, P<0.0001$; Fig. $3 \mathrm{~g}$ ). If only observations with traveling time are considered, both the left-right count and the fore-aft count show a weak response to traveling time (Figs. $3 \mathrm{~d}$ and 3e). However, the same range of motion sensor counts occurred when there

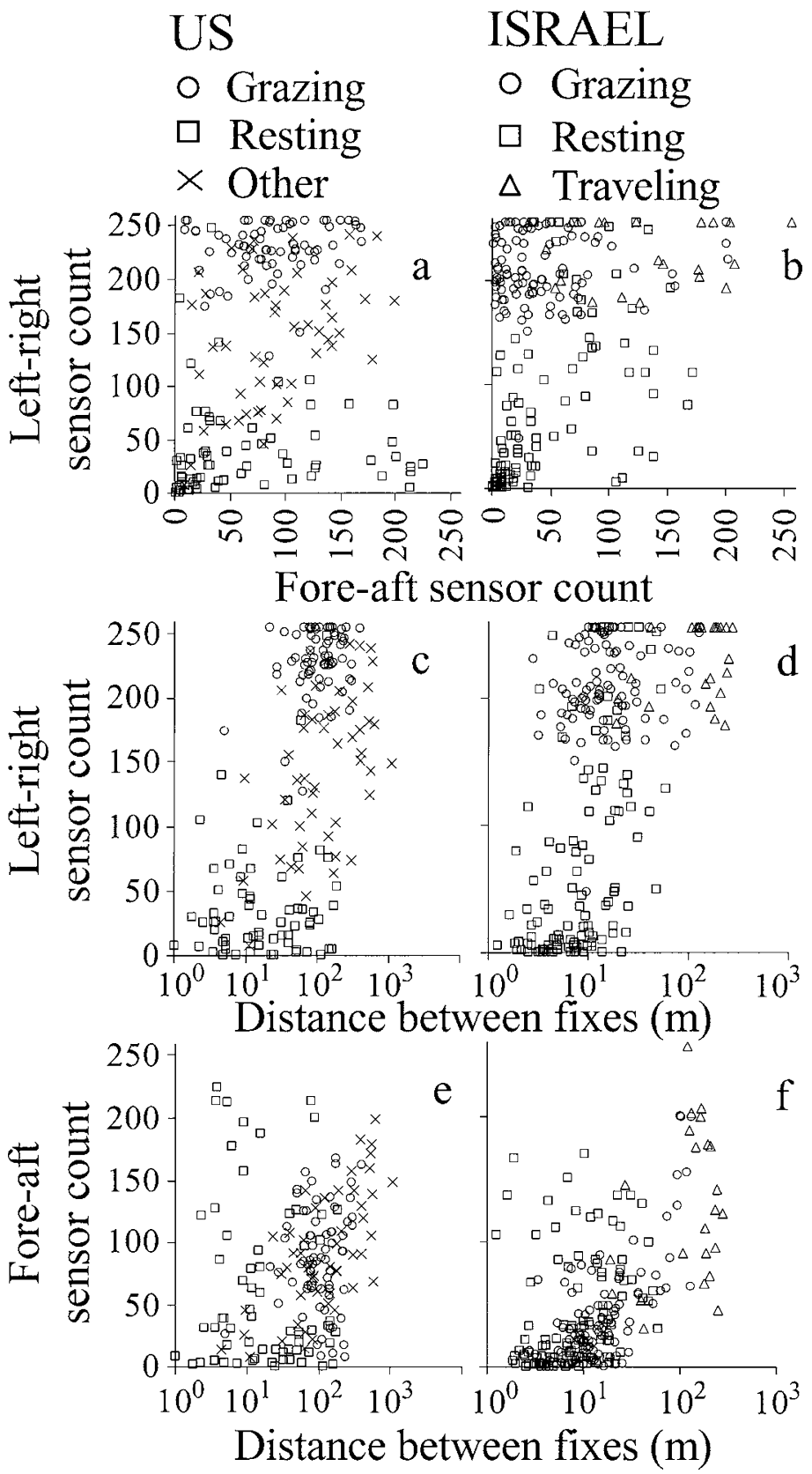

Figure 2. Relationships between each pair of the 3 collar variables: the left-right sensor count, the fore-aft sensor count, and the distance between GPS fixes. For the US data, different symbols are used for observations that predominantly (at least 16 out of 20 minutes) recorded grazing, resting, and all other observations. For Israel, activities are for an entire 5-minute observation. A logarithmic scale is used for Distance to improve separation at low Distance. Sensor count summations are for 3-minute (US) and 4-minute (Israel) periods.

was no traveling. When all data were included, the correlation coefficients for the relationships between traveling time and left-right count and fore-aft count were only $0.13(P=0.0742)$ and $0.34(P<0.0001)$, respectively.

Figure 4 shows the frequency distributions of the Israeli data on the 3 collar variables, as related to observed animal activity. Lying and standing appear to evoke similar responses, and would be difficult to separate in a predictive model. More 

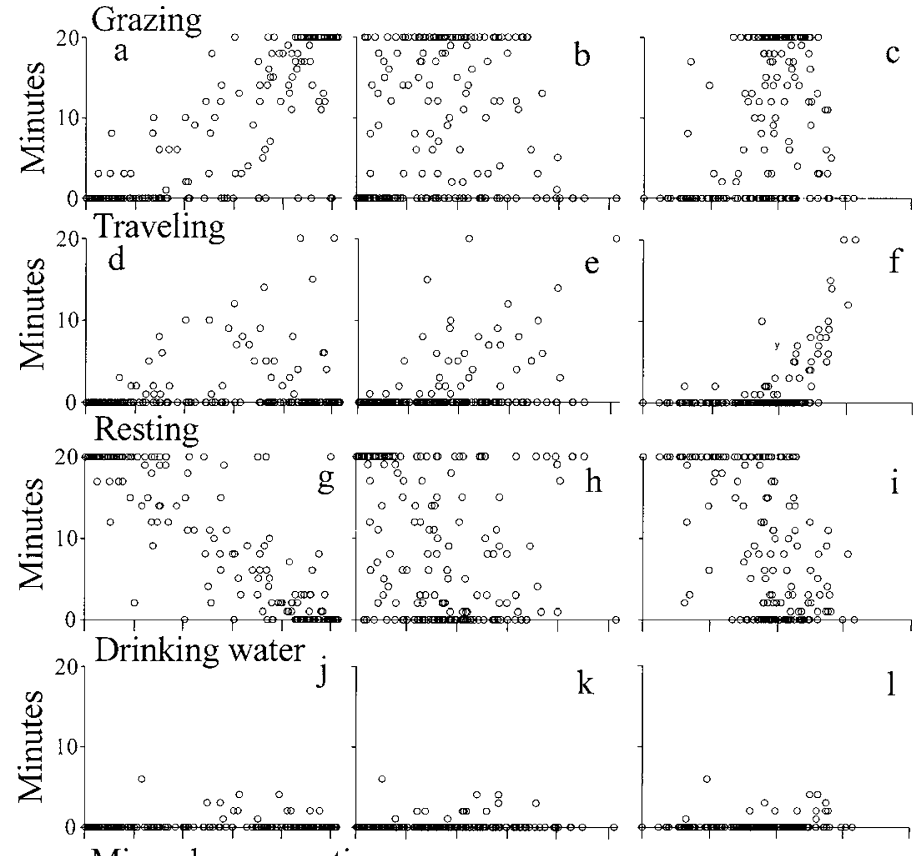

$\mathrm{k}$

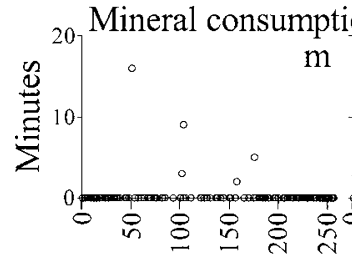

Left-right

sensor count

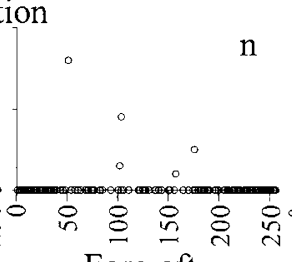

Fore-aft

sensor count
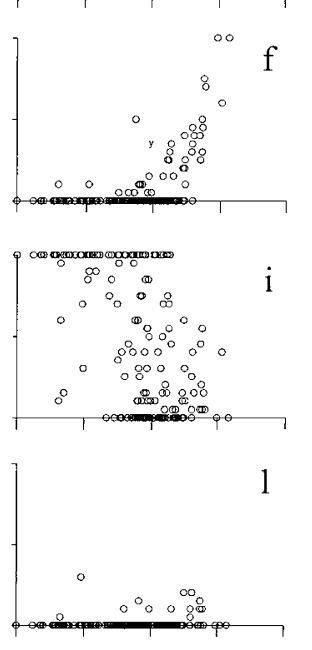

1

Figure 3. Relationships for the US data between the number of minutes of a 20-minute observation dedicated to each animal activity and the 3 collar variables: the left-right sensor count, the fore-aft sensor count, and the distance between GPS fixes. Rest is the pooling of standing and lying.

importantly, a traveling activity that did not cover a long distance $(>50 \mathrm{~m})$ would be difficult to separate from grazing. An uninterrupted bout of 5 minutes of grazing almost invariably resulted in a reading of the left-right motion counter exceeding 150 , with a fairly uniform distribution over the range 150-255. This left-right count range was also reached, albeit rarely, during 5 -minute periods of standing or lying.

\section{United States}

Multiple regression analysis of the number of minutes dedicated to grazing during a 20-minute observation yielded a simple model (Table 2; Eq. 1) that accounted for $81 \%$ of the variation in grazing time.

Forward stepwise regression analysis for grazing time, allowing the model to select from basic and computed variables, added CountLeftRight/CountForeAft and CountLeftRight/Distance to the above 2 variables (Table 2; Eq. 2). The final model achieved only a slight improvement in $R^{2}(84 \%)$.

The corresponding equations for resting time and traveling time provided by forward stepwise regression accounted for $84 \%$ and $74 \%$ of the variation, respectively (Table 2; Eqs. 3 and 4).

The predicted times for grazing, resting, and traveling were less than zero for $20 \%, 13 \%$, and $31 \%$ of observations,

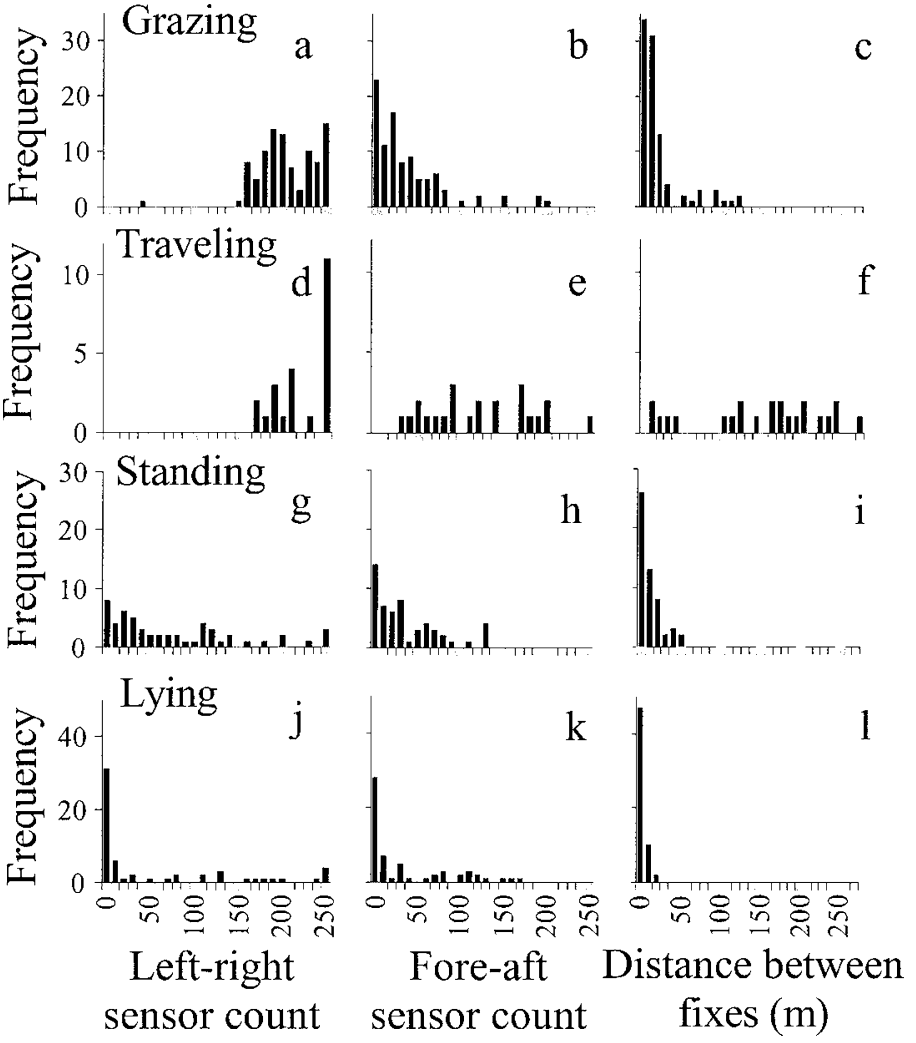

Figure 4. Frequency distributions for the Israel data of the 3 collar variables: the left-right sensor count, the fore-aft sensor count, and the distance between GPS fixes, according to animal activity. Frequency is the number of 5 -minute observations, out of a total of 231.

respectively. For observations that were entirely accounted for by grazing, resting, and traveling $(n=178)$, the sum of the predicted times ranged from 16.6 to 19.8 minutes, with a tight distribution about the mean of 19.6 minutes.

Because a different collar was used for each cow observed in the United States, we can examine the cow and collar effects in combination. Cow/collar was not significant $(P=0.07)$ when included in the analysis of variance of grazing time. Multiple regression analysis of grazing time for each cow/collar separately yielded a wide range of goodness of fit, accounting for between $72 \%$ and $92 \%$ of the variation. Nevertheless, the coefficients of the prediction formula for each cow/collar were broadly similar to those of the global model cited above. Cow/collar was significant $(P=0.04)$ in the analysis of variance of traveling time. In regression analysis of traveling time for each cow/collar separately, Distance was highly significant $(P<0.0001)$ in all cases, whereas CountLeftRight was not $(P>0.22)$. Cow/collar was also significant $(P=0.02)$ in the analysis of variance of resting time.

The distance between GPS locations (Distance) alone, in linear and polynomial models, was found to be a very poor indicator of grazing and resting time. For example, third-order polynomial models accounted for $24 \%$ and $30 \%$ of the variation in grazing time and resting time, respectively. The Distance variable is a better indicator of traveling time, and a thirdorder polynomial model accounted for $75 \%$ of the variation. Note that no traveling time was recorded for $81 \%$ of the observations. 
Table 2. Equations obtained by regression analysis (US data) and discriminant analysis (Israel data) of Lotek collar data. For the US data, the formula predicts the number of minutes dedicated to the specified activity during a 20 -min observation. For the Israel data, a single activity is predicted for a 5-min observation, on the basis of the function yielding the minimum value.

\begin{tabular}{|c|c|c|c|c|c|c|}
\hline Eqn. & Data Source & Analyses & Dependent Variable & Formula $^{1}$ & SE & $R^{2}$ \\
\hline 1 & United States & Multiple regression & Grazing time & $=0.091 \mathrm{CLR}-0.015$ Distance -1.58 & 3.84 & $81 \%$ \\
\hline 2 & United States & Forward stepwise regression & Grazing time & $\begin{aligned}= & 0.094 \text { CLR }-0.017 \text { Distance }-0.075 \\
& \text { CLR/CFA }-0.11 \text { CLR/Distance }-0.96\end{aligned}$ & 3.54 & $84 \%$ \\
\hline 3 & United States & Forward stepwise regression & Resting time & $\begin{aligned}= & 20.6-0.086 \mathrm{CLR}+0.084 \\
& \mathrm{CLR} / \mathrm{CFA}+0.070 \mathrm{CLR} / \text { Distance }\end{aligned}$ & 3.43 & $84 \%$ \\
\hline 4 & United States & Forward stepwise regression & Traveling time & $\begin{aligned}= & 0.16+0.017 \text { Distance }-0.0072 \\
& \text { CLR }-0.027 \text { Distance } / \text { CFA }\end{aligned}$ & 1.69 & $74 \%$ \\
\hline 5 & Israel & Discriminant & Activity: Grazing & $\begin{array}{l}\text { F[grazing]: }-1.1976 \times 10^{-1} \mathrm{CLR}+ \\
2.7003 \times 10^{-2} \mathrm{CFA}-2.5194 \times 10^{-2} \\
\quad \text { Distance }+12.2669\end{array}$ & $\mathrm{n} / \mathrm{a}$ & $n / a$ \\
\hline 6 & & & Resting & $\begin{array}{l}\text { F[resting]: }-3.0737 \times 10^{-2} \mathrm{CLR}- \\
2.17768 \times 10^{-2} \mathrm{CFA}- \\
8.6746 \times 10^{-5} \text { Distance }+ \\
1.4749\end{array}$ & & \\
\hline 7 & & & Traveling & $\begin{array}{l}F\left[\text { traveling]: }-1.0121 \times 10^{-1} \mathrm{CLR}+\right. \\
\quad 1.4442 \times 10^{-3} \text { CFA }- \\
2.7255 \times 10^{-1} \text { Distance }+ \\
31.9121\end{array}$ & & \\
\hline
\end{tabular}

${ }^{1}$ CLR indicates CountLeftRight; CFA, CountForeAft.

The prediction of grazing time by regression tree analysis, allowing the routine to select only from the 3 basic collar variables, yielded the following series of split points:

1. CountLeftRight $=183$. This single split created two predicted values: 2.4 minutes of grazing if CountLeftRight $<183(n=109)$ and 17.5 minutes of grazing if CountLeftRight $\geq 183(n=86)$. Overall $R^{2}=72 \%$.

2. Distance $=586$ within CountLeftRight $\geq 183$. The second split was somewhat sensitive to the minimum cell size permitted in the analysis, this result being obtained with no limitation. This split separated three observations for which Distance was greater than 586 . Overall $R^{2}=78 \%$.

3. CountLeftRight $=110$ within CountLeftRight $<183$. Overall $R^{2}=84 \%$.

4. Distance $=395$ within $110<$ CountLeftRight $<183$. Overall $R^{2}=85 \%$.

The four-split solution is shown graphically in Figure 5, together with the predicted grazing time for each sector of the space created. It is only with the subsequent split that CountForeAft entered the model, acting on a small grouping of 19 observations (not shown). Allowing the regression tree to select from both the computed and the basic variables did not materially improve predictive ability for the same number of splits. The CountLeftRight variable occupied the same central role in the tree as it did when the computed variables were not included.

Although the two regression approaches yielded models with similar coefficients of determination, an examination of residuals indicated an important difference between approaches. Regression tree analysis predicted a low grazing time of 0.9 minute for $85 \%$ of the 80 observations that contained no grazing minutes at all. It predicted a high grazing time of 18.1 minutes for all but one of the 56 observations that were entirely devoted to grazing. The predictive power was weaker for the smaller group of observations that had intermediate levels of grazing. Overall, the absolute difference between observed and

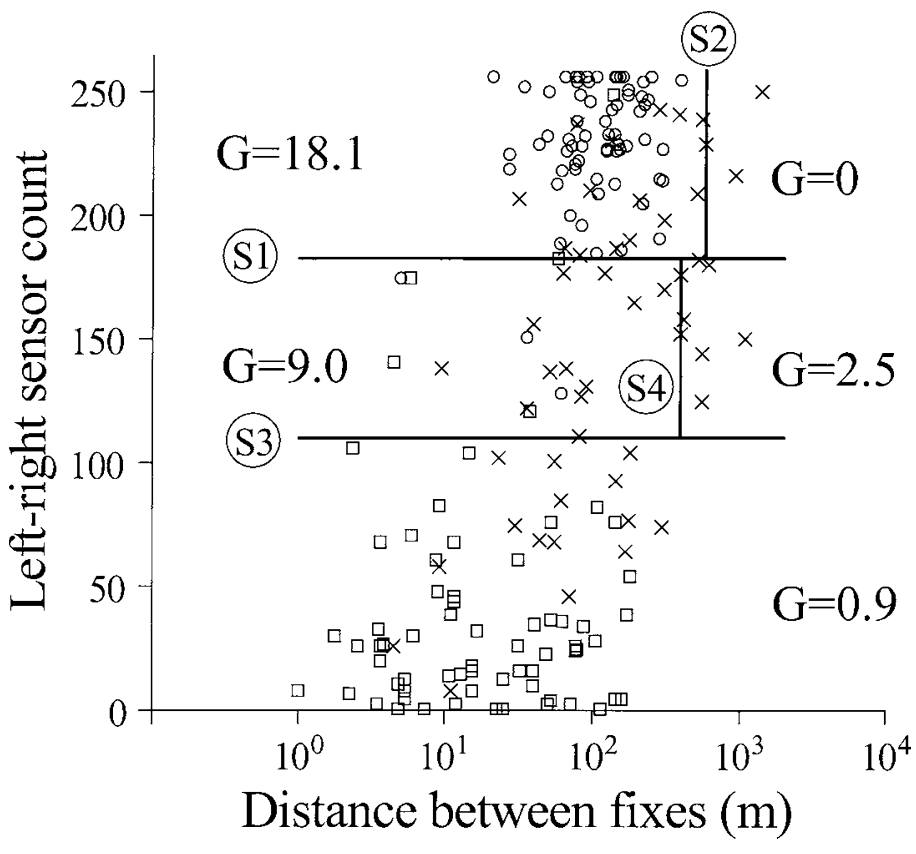

Figure 5. Distribution of the US observations according to the left-right sensor count and the distance between GPS fixes, and the split lines generated by regression tree analysis, numbered $(\mathrm{S} 1, \mathrm{~S} 2, \ldots)$ in order of entry into the model. $G$ indicates the predicted grazing time (number of minutes out of 20) for each sector delineated by the split lines. The observed animal activity is indicated by the symbol: $\bigcirc$, predominantly grazing; $\square$, predominantly resting; $\times$, other. 
Table 3. Frequency of observed vs. predicted activity during 5-min observations (Israel data) on the basis of discriminant analysis. Factors in the model were the left-right and fore-aft motion sensor counts and the straight-line distance between GPS locations. Standing and lying activities were treated separately, ${ }^{2}$ or together as resting. ${ }^{3}$

\begin{tabular}{|c|c|c|c|c|}
\hline \multirow{2}{*}{$\begin{array}{l}\text { Observed } \\
\text { Activity }\end{array}$} & \multicolumn{4}{|c|}{ Predicted Activity } \\
\hline & $G$ & $\mathrm{~T}$ & $S$ & $\mathrm{~L}$ \\
\hline$\overline{G^{1,2}}$ & 87 & 7 & 0 & 1 \\
\hline $\mathrm{T}^{2}$ & 5 & 18 & 0 & 0 \\
\hline$S^{2}$ & 9 & 0 & 15 & 30 \\
\hline \multirow[t]{2}{*}{$L^{2}$} & 7 & 0 & 7 & 45 \\
\hline & G & & & $\mathrm{R}$ \\
\hline $\mathrm{G}^{3}$ & 87 & & & 1 \\
\hline $\mathrm{T}^{3}$ & 5 & & & 0 \\
\hline $\mathrm{R}^{3}$ & 19 & & & 94 \\
\hline
\end{tabular}

${ }^{1} \mathrm{G}$ indicates grazing; $\mathrm{T}$, traveling; $\mathrm{S}$, standing; L, lying; $\mathrm{R}$, resting $(\mathrm{S}+\mathrm{L})$

${ }^{2}$ Standing and lying activities were treated separately. Misclassifications: 66 of $231(29 \%)$.

${ }^{3}$ Standing and lying activities were treated together as resting. Misclassifications: 32 of $231(14 \%)$.

predicted grazing time was $<2.5$ minutes and $<5$ minutes for $80 \%$ and $87 \%$ of observations, respectively. In contrast, classical regression analysis predicted a range of grazing times for observations that were, in fact, all or nothing. This approach performed better for grazing times within the intermediate range. For regression, the absolute difference between observed and predicted grazing time was $<2.5$ minutes and $<5$ minutes for $67 \%$ and $88 \%$ of observations, respectively.

Regression tree analysis of resting time resulted in 3 successive splits of CountLeftRight, accounting for $85 \%$ of the variation. The four regions of CountLeftRight were: CountLeftRight $\leq 50, \quad 50<$ CountLeftRight $\leq 121, \quad 121<$ CountLeftRight $\leq 188$, CountLeftRight $>188$, and the corresponding predicted resting times were 1.0, 7.7, 15.3, and 19.6 minutes, respectively.

The first 3 splits in the analysis of traveling time were based on a single variable, Distance, and accounted for $80 \%$ of the variation. The 4 regions of Distance were: Distance $\leq 168,168<$ Distance $\leq 395,395<$ Distance $\leq 586$, Distance $>586$, and the corresponding predicted traveling times were 0.2, 2.0, 7.8, and 16.2 minutes, respectively. At the fourth split, the region defined by $168<$ Distance $\leq 395$ was subdivided according to CountLeftRight, using a threshold of 190 , and this model accounted for $83 \%$ of the variation in traveling time. Regression tree analysis based only on Distance yielded very poor results for grazing and resting time.

Predicted values of grazing, resting, and traveling times by regression tree analysis were never less than zero. However, the sum of the predicted times could be greater than 20 minutes (range 16.4-26.9 minutes), with a multimodal and dispersed distribution about its mean of 19.7 minutes.

\section{Israel}

Discriminant analysis of animal activity (grazing, traveling, standing, lying) based only on the basic collar variables CountLeftRight, CountForeAft, and Distance, yielded a mis-

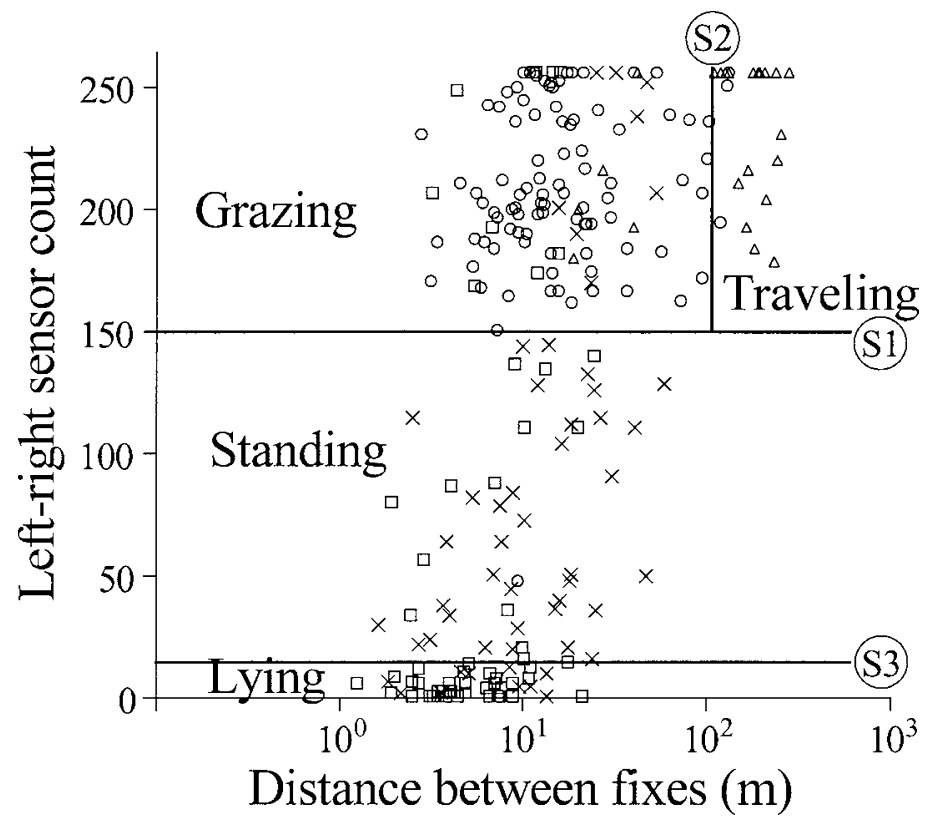

Figure 6. Distribution of the Israel observations according to the leftright sensor count and the distance between GPS fixes, and the split lines generated by classification tree analysis, numbered $(\mathrm{S} 1, \mathrm{~S} 2, \ldots)$ in order of entry into the model. The predicted animal activity is indicated within each sector delineated by the split lines. A nonconsequential split according to the fore-aft sensor count is not indicated. The observed animal activity is indicated by the symbol: $\bigcirc$, grazing; $\triangle$, traveling; $\times$, standing; $\square$, lying.

classification rate of $29 \%$ (Table 3; Wilks' Lambda $=0.1526$, $P<0.0001$ ). Almost all grazing observations were correctly classified, but other activities were sometimes misclassified as grazing. Little advantage was gained when computed products and ratios of CountLeftRight, CountForeAft, and Distance were added to the model. When standing and lying activities were combined as resting, the model containing the factors CountLeftRight, CountForeAft, and Distance yielded a misclassification rate of $14 \%$ (Table 3; Wilks' Lambda $=0.1573$, $P<0.0001)$. The discriminant functions for the latter analysis are given in Equations 5-7 of Table 2. The function yielding the minimum value for an observation determined the predicted activity.

Classification tree analysis of the 4 animal activities (grazing, traveling, standing, lying), allowing the routine to select only from the 3 basic collar variables, yielded the following series of split points (see Fig. 6):

1. CountLeftRight $=150$. Observations for which Count LeftRight $<150(n=96)$ had probabilities of $51 \%$ and $48 \%$ of recording lying or standing, respectively. Observations for which CountLeftRight $\geq 150(n=135)$ had a probability of $70 \%$ of recording grazing.

2. Distance $=106.5$ within CountLeftRight $\geq 150$. Observations for which Distance $>106.5(n=21)$ were associated with traveling, and the remainder with grazing $(n=114)$.

3. CountForeAft $=30$ within CountLeftRight $\geq 150$ and Distance $<106.5$ (not shown in Fig. 6). Both subdivisions led to a prediction of grazing based on the activity 
with the highest probability; however, the 50 observations associated with CountForeAft $<30$ all recorded grazing, whereas the 64 observations for which CountFore Aft $\geq 30$ had a probability of $64 \%$ of recording grazing.

4. CountLeftRight $=15$ within CountLeftRight $<150$. The 46 observations for which CountLeftRight $<15$ are most likely to record lying down, whereas the 50 observations for which CountLeftRight $\geq 15$ are most likely to record standing.

Overall, this model misclassified 50 of 231 observations, or $22 \%$ of the observations (Table 4 ).

When lying down and standing were combined as resting, the same tree as above was obtained. The only difference was that the group of 96 observations created by the first split (CountLeftRight $<150$ ) comprised almost entirely $(99 \%)$ resting and did not split. Adding computed values to the analyses did not noticeably improve predictions of activity. Grouping standing and lying resulted in 27 misclassifications, or $12 \%$ of the observations (Table 4 ). In both this and the previous model, 91 of the 95 cases of grazing were correctly classified, though other activities were misclassified as grazing.

The only activity that can be inferred with some degree of reliability solely on the basis of Distance is traveling. Classification tree analysis separated out 18 of the 23 observations of traveling with a single split at $107 \mathrm{~m}$.

\section{DISCUSSION}

Qualitatively similar results were obtained in both studies. Distance data alone were not useful for predicting grazing and resting. Motion sensor data greatly improved our predictive ability for these activities, as well as for traveling.

Turner et al. (2000) examined the deployment of Lotek GPS collars on cattle; they used the same operational schedule as was used in Israel in the present study (GPS fix interval $=5$ minutes and activity-sampling period $=4$ minutes). They reported very high accuracy of classification of grazing and resting activities, with a threshold of 200 for the sum of the two sensor counts. In the case of the Israeli grazing and resting observations, the summed counts of the left-right and fore-aft sensors yielded a misclassification rate of $20 \%$, whereas the left-right count alone performed better, with a misclassification rate of $10 \%$.

The misclassifications obtained in the discriminant analysis and classification tree analysis of the Israel data were not evenly distributed. The misclassification of resting as grazing was the main source of error. We therefore advise caution in the interpretation of grazing "hot-spots" on utilization maps derived from GPS collar data. If possible, it should be verified that these are not resting areas.

Complete accuracy in activity determinations is difficult to achieve for several reasons: 1) There is an indirect connection between the independent and dependent variables, and there are many potential sources of noise in the data. 2) Resting activity is not always associated with zero or very low motion sensor counts (Figs. 3 and 4), as found by Moen et al. (1996a) using Lotek GPS collars on moose. We would expect that movement of the head and neck while the animal is considered to be resting (e.g., comfort movements, grooming, or respond-
Table 4. Frequency of observed vs. predicted activity during a 5-min period (Israel data) on the basis of classification tree analysis. Factors in the model were the left-right and fore-aft motion sensor counts and the straight-line distance between GPS locations.

\begin{tabular}{lrrrr}
\hline Observed & \multicolumn{4}{c}{ Predicted Activity } \\
\cline { 2 - 5 } Activity & $\mathrm{G}$ & $\mathrm{T}$ & $\mathrm{S}$ & $\mathrm{L}$ \\
\hline $\mathrm{G}^{1,2}$ & 91 & 3 & 1 & 0 \\
$\mathrm{~T}^{2}$ & 5 & 18 & 0 & 0 \\
$\mathrm{~S}^{2}$ & 8 & 0 & 36 & 10 \\
$\mathrm{~L}^{2}$ & 10 & 0 & 13 & 36 \\
& & Predicted Activity & \\
\cline { 2 - 5 } & $\mathrm{G}$ & $\mathrm{T}$ & $\mathrm{R}$ \\
\hline $\mathrm{G}^{3}$ & 91 & 3 & 1 \\
$\mathrm{~T}^{3}$ & 5 & 0 & 0 \\
$\mathrm{R}^{3}$ & 18 & & 0 & \\
\hline
\end{tabular}

${ }^{1} \mathrm{G}$ indicates grazing; $\mathrm{T}$, traveling; $\mathrm{S}$, standing; $\mathrm{L}$, lying; $\mathrm{R}$, resting $(\mathrm{S}+\mathrm{L})$.

${ }^{2}$ Standing and lying activities were treated separately. Misclassifications: 50 (22\%).

${ }^{3}$ Standing and lying activities were treated together as resting. Misclassifications: 27 (12\%).

ing to insect pests) may be registered by the sensors, but more detailed observations would be required to verify this possibility. 3) Walking is common to both grazing and traveling. 4) The pattern of head and neck movement, and, therefore, of motion sensor counts, associated with active grazing may not be the same for all animals or sward structures. 5) GPS collars may differ in motion sensitivity, because of differences among animals in their fittings, which may affect how neck movements are translated into motion counts (Moen et al. 1996a; Turner et al. 2000). 6) Meandering of the foraging path of the animal will tend to blur the relationship between grazing time and distance traveled. 7) Not all minutes between GPS location fixes are represented in the motion sensor counts. 8) Short-lived changes of activity during an observation interval were ignored. Lower error rates in the determination of animal activity might be expected from the use of leg and jaw sensors in conjunction with GPS, if it were technically feasible.

We were surprised that the distance measurements did not facilitate a more definitive separation between resting and grazing. This problem may be partially due to the limits of GPS accuracy. The relatively short GPS integration times used by the Lotek receivers (to conserve energy) may come at a cost of accuracy, even with differential correction. Six tests of accuracy were conducted in the United States after selective availability was disabled, using stationary Lotek collars (2200 series; Ganskopp, unpublished data, 2004). In February/March 2004, 3 collars were configured to integrate 1000 GPS positions at 5 -minute intervals and 3 collars programmed to integrate 1000 positions at 20-minute intervals. Differences between collars or integration intervals were small. For the pooled data ( $n=5994)$, the 97.5 percentile, median, and mean of the distance between adjacent fixes in time were 9.3, 2.1 , and $2.7 \mathrm{~m}$, respectively, for the uncorrected data. After differential correction, corresponding values were $5.8,1.7$, and $2.1 \mathrm{~m}$, respectively.

How does this "stationary distance" produced by GPS error compare with those covered in traveling and grazing? The speed of travel during pure traveling activity (assuming a straight line of travel) can be estimated from the US data by 
considering only observations with nonzero traveling time $(n=37)$. Linear regression between distance and traveling time yielded an intercept of -5 (not significant) and slope of 54 $\mathrm{m} \mathrm{min}{ }^{-1}$. For the Israeli data, there were 23 cases of traveling (for an entire 5-minute observation period) with a mean distance of $150 \mathrm{~m}$, i.e., $30 \mathrm{~m} \mathrm{~min}^{-1}$. Similarly, the speed of travel during grazing can be estimated from the US data by considering observations that recorded only grazing (possibly with unrecorded interludes of traveling) $(n=56)$. The mean distance covered during these observations was $124 \mathrm{~m}$, i.e., $6 \mathrm{~m}$ $\min ^{-1}$. For the Israel data, there were 95 cases of grazing, with a mean distance of $24 \mathrm{~m}$, i.e., about $5 \mathrm{~m} \mathrm{~min}^{-1}$. Thus, distance measurements are expected to assist more in the separation of resting and traveling activities than in the separation of resting and grazing activities.

Better predictive models would be expected for a GPS fix interval of 5 minutes than for one of 20 minutes because a single activity is more likely for each observation. However, GPS error may hamper the separation of resting and grazing on the basis of distance at the shorter time scale. If bouts of grazing and resting tend to span several time steps, then the distance to earlier GPS location fixes, and not just the most recent, deserves future examination as a means of differentiating grazing and resting. Further testing of the predictive equations developed in this study will require new sources of synchronized observer and collar data.

The two most obvious reasons for elucidating cattle activities from GPS collars are a need to quantify activity budgets or travels of animals exposed to differing treatments (Ganskopp 2001; Schauer 2003), and a desire to relate georeferenced activities to site-specific characteristics of the habitat via a geographic information system. Vibracorders (Stobbs 1970) were the first instruments used to quantify grazing times of cattle in remote environments, but such data have a complete lack of spatial association. Similarly, pedometers have successfully been used to measure travels of cattle (Anderson and Kothmann 1977), but again, data have no spatial characteristics, and animals must be bipedometered with units calibrated to compensate for individual unit/animal bias (Anderson and Urquhart 1986). Automated telemetry systems involving triangulation algorithms have increased the frequency with which one can monitor animal location, but such units are still plagued with levels of spatial error approaching $50 \mathrm{~m}$ (Findholt et al. 1996). Although GPS collars that determine positions at scheduled intervals will most certainly underestimate an animal's travels, the integration of motion sensors and accurate position data provide the best method available to date for determinations of animal activity and resource use in remote environments.

\section{MANAGEMENT IMPLICATIONS}

Grazing, traveling, and resting activities of free-ranging cattle can be inferred with reasonable accuracy from data provided by Lotek GPS collars. Considered on its own, the distance between adjacent GPS fixes was not a good indicator of animal activity during the corresponding time interval, but combining motionsensor data, especially along the left-right axis of movement, with the distance data greatly improved the prediction of animal activity. The inclusion of products and ratios of the basic collar variables in the statistical models did not markedly enhance predictive ability. When the interval between GPS fixes was short ( 5 minutes), animal activity was defined categorically, and predicted by means of discriminant analysis or classification tree analysis, with a misclassification rate of $12 \%-14 \%$. The main source of error in tree-based analysis was the misclassification of resting as grazing. For long intervals between GPS fixes (20 minutes), the proportion of time allocated to any one activity can be predicted by models based on multiple linear regression or regression tree analysis that account for $80 \%-$ $85 \%$ of the variation.

\section{ACKNOWLEDGMENTS}

Authors gratefully acknowledge the assistance of Chava Aharon and Yehuda Yehuda with the field work, and of Orit Israelson with the processing of GPS and GIS data.

\section{LITERATURE CITED}

Adrados, C., I. Girard, J. P. Gendner, and G. Janeau. 2002. Global positioning system (GPS) location accuracy improvement due to selective availability removal. Comptes Rendus Biologies 325:165-170.

Anderson, D. M., And M. M. Kothmann. 1977. Monitoring animal travel with digital pedometers. Journal of Range Management 30:316-317.

AndeRson, D. M., AND N. S. UrouhaRt. 1986. Using digital pedometers to monitor travel of cows grazing arid rangeland. Applied Animal Behaviour Science 16:11-23.

Biggs, J. R., K. D. Bennett, and P. R. Fresquez. 2001. Relationship between home range characteristics and the probability of obtaining successful global positioning system (GPS) collar positions for elk in New Mexico. Western North American Naturalist 61:213-222.

Blake, S., I. Douglas Hamilton, and W. B. Karesh. 2001. GPS telemetry of forest elephants in Central Africa: results of a preliminary study. African Journal of Ecology 39:178-186.

Blanc, F., and A. BreluRut. 1997. Short-term behavioral effects of equipping red deer hinds with a tracking collar. Zeitschrift fur Saugetierkunde-International Journal of Mammalian Biology 62:18-26.

Bowman, J. L., C. O. Kochanny, S. Demarais, and B. D. Leopold. 2000. Evaluation of a GPS collar for white-tailed deer. Wildlife Society Bulletin 28:141-145.

Breiman, L., J. H. Friedman, R. A. Olshen, and C. J. Stone. 1984. Classification and regression trees. Belmont, CA: Wadsworth. $358 \mathrm{p}$.

Coughenour, M. B. 1991. Spatial components of plant-herbivore interactions in pastoral, ranching, and native ungulate ecosystems. Journal of Range Management 44:530-542.

Daнms, S. 2004. Combination of variance components estimation, regression trees and logistic regression as a concept for exploring associations between animal husbandry conditions and lesions found at slaughter. Deutsche Tierarztliche Wochenschrift 111:178-181.

Del Cima, R., M. F. D'Antuono, and W. K. Anderson. 2004. The effects of soil type and seasonal rainfall on the optimum seed rate for wheat in Western Australia. Australian Journal of Experimental Agriculture 44:585-594.

Dussault, C., R. Courtols, J.-P. Ouellet, and J. Huot. 1999. Evaluation of GPS telemetry collar performance for habitat studies in the boreal forest. Wildlife Society Bulletin 27:965-972.

Findholt, S. L., B. K. Johnson, L. D. Bryant, and J. W. Thomas. 1996. Corrections for position bias of a Loran-C radio-telemetry system using DGPS. Northwest Science 70:273-280.

GANSKOPP, D. 2001. Manipulating cattle distribution with salt and water in large arid-land pastures: a GPS/GIS assessment. Applied Animal Behaviour Science 73:251-262. 
Girard, I., J. P. Ouellet, R. Courtols, C. Dussault, and L. Breton. 2002. Effects of sampling effort based on GPS telemetry on home-range size estimations. Journal of Wildlife Management 66:1290-1300.

Graham, C. 2001. Habitat selection and activity budgets of Keel-billed Toucans at the landscape level. Condor 103:776-784

Gutman, M., Z. Holzer, N. G. Seligman, And I. Noy-Meir. 1990. Stocking density and production of a supplemented beef herd grazing yearlong on Mediterranean grassland. Journal of Range Management 43:535-539.

Gutman, M., And N. G. Seligman. 1979. Grazing management of Mediterranean foothill range in the upper Jordan river valley. Journal of Range Management 32:86-92.

Hulbert, I. A. R., AND J. French. 2001. The accuracy of GPS for wildlife telemetry and habitat mapping. Journal of Applied Ecology 38:869-878.

Hulbert, I. A. R., J. T. B. Wyllie, A. Waterhouse, J. French, and D. McNulty. 1998. A note on the circadian rhythm and feeding behaviour of sheep fitted with a lightweight GPS collar. Applied Animal Behaviour Science 60:359-364.

Johnson, C. J., D. C. Heard, and K. L. Parker. 2002a. Expectations and realities of GPS animal location collars: results of three years in the field. Wildlife Biology 8:153-159.

Johnson, C. J., K. L. Parker, D. C. Heard, and M. P. Gillingham. 2002b. Movement parameters of ungulates and scale-specific responses to the environment. Journal of Animal Ecology 71:225-235.

Merrill, S. B., L. G. Adams, M. E. Nelson, and L. D. Mech. 1998. Testing releasable
GPS radiocollars on wolves and white-tailed deer. Wildlife Society Bulletin 26:830-835.

Moen, R., J. Pastor, and Y. Cohen. 1996a. Interpreting behavior from activity counters in GPS collars on moose. Alces 32:101-108.

Moen, R., J. Pastor, Y. Cohen, and C. C. Schwartz. 1996b. Effects of moose movement and habitat use on GPS collar performance. Journal of Wildlife Management 60:659-668.

Rempel, R. S., A. R. Rodgers, and K. F. Abraham. 1995. Performance of a GPS animal location system under boreal forest canopy. Journal of Wildlife Management 59:543-551.

Rodgers, A. R., AND P. Anson. 1994. Animal-borne GPS: tracking the habitat. GPS World 5:20-32.

Rutter, S. M., N. A. Beresford, and G. Roberts. 1997. Use of GPS to identify the grazing areas of hill sheep. Computers and Electronics in Agriculture 17: 177-188.

SAS InstituTE. 2002. JMP Statistics and Graphics Guide, Version 5. Cary, NC. 707 p.

SCHAUER, C. S. 2003. Influence of protein supplementation frequency on cows consuming low-quality forage: performance, grazing behavior, and variation in supplement intake [thesis]. Corvallis, OR: Oregon State University. 108 p.

Stoввs, T. H. 1970. Automatic measurement of grazing time by dairy cows on tropical grass and legume pastures. Tropical Grasslands 4:237-244.

Turner, L. W., M. C. Udal, B. T. Larson, and S. A. Shearer. 2000. Monitoring cattle activity and pasture use with GPS and GIS. Canadian Journal of Animal Science 80:405-413. 